\title{
Cost-effective Management of Severe Gastroesophageal Reflux Disease: Toward an Improved Understanding of Anti-reflux Surgery
}

\author{
Chan Hyuk Park \\ Department of Internal Medicine, Hanyang University Guri Hospital, Hanyang University College of Medicine, Guri, Gyeonggi-do, Korea
}

\author{
Article: Anti-reflux surgery versus proton pump inhibitors for severe gastroesophageal reflux disease: a cost-effectiveness \\ study in Korea \\ Park S, Park JM, Park JM, et al \\ (J Neurogastroenterol Motil 2020;26:215-223)
}

Gastroesophageal reflux disease (GERD) can generally be well-managed using proton pump inhibitors (PPI). ${ }^{1}$ Four weeks of PPI therapy can achieve about $70 \%$ of healing rate and $60-90 \%$ of complete symptom resolution. ${ }^{2}$ However, GERD is a great socioeconomic burden. In the United States, the direct cost of managing GERD exceeds $\$ 12$ billion, which is higher than the overall direct cost from all digestive tract cancers ( $\$ 8.4$ billion). ${ }^{3}$ The significant cost involved in the management of patients with GERD is caused by a high prevalence in the population and the need for long-term medications. ${ }^{4,5}$

Recently, the interest in managing GERD surgically has increased. ${ }^{6-8}$ A recent randomized-controlled trial showed that laparoscopic Nissen fundoplication was superior to medication in reducing reflux-related heartburn. ${ }^{6}$ In the study, treatment success, which was defined as an improvement of $50 \%$ or more in the GERDHealth Related Quality of Life score, was achieved in 67\% of patients who underwent surgery, while $28 \%$ of patients who received active medical therapy including omeprazole and baclofen achieved treatment success. ${ }^{6}$ In another recent study in an Asian population, laparoscopic Nissen fundoplication was shown to be effective in patients for whom medical treatment was ineffective or who were unable to discontinue medication due to symptom recurrence. Although medication should be the first-line treatment for patients with GERD and even though a large proportion of patients can be medically managed, anti-reflux surgery may offer an option for selected patients. ${ }^{6}$

The efficacy of anti-reflux surgery in the management of GERD patients is well-recognized. However, cost-effectiveness must be a primary consideration before recommending surgical treatment widely in clinical practice. In this issue of the Journal of Neurogastroenterology and Motility, Park et al ${ }^{9}$ reported a costeffective analysis between anti-reflux surgery and medication in patients with severe GERD in Korea. They showed that the surgical approach resulted in cost savings of $\$ 551$ per patient and that the quality-adjusted life years had a gain of 1.18 compared to medication among patients with severe GERD over a 10-year period.

Received: February 11, 2020 Revised: None Accepted: February 20, 2020

(ङ) This is an Open Access article distributed under the terms of the Creative Commons Attribution Non-Commercial License (http:// creativecommons. org/licenses/by-nc/4.0) which permits unrestricted non-commercial use, distribution, and reproduction in any medium, provided the original work is properly cited.

*Correspondence: Chan Hyuk Park, MD, PhD Department of Internal Medicine, Hanyang University Guri Hospital, Hanyang University College of Medicine, 153 Gyeongchun-ro, Guri, Gyeonggi-do 11923, Korea

Tel: +82-31-560-2230, Fax: +82-31-553-7369, E-mail: yesable7@gmail.com 
Although the initial cost of surgical treatment was higher than that of medication, the average cost of anti-reflux surgery decreased as the follow-up period increased. In the study, the break-even point for anti-reflux surgery over medication was estimated to be 9 years. However, the results should be interpreted with caution because the cost-effectiveness was largely estimated on assumptions. In the study, severe GERD was defined as having symptoms, including heartburn and regurgitation, that require either a continuous double dose of PPI or surgical treatment. This sample population only represented an extremely small proportion of GERD patients in the general population, because double dosage medication is not approved for GERD in most PPIs in Korea. In other words, most patients with severe GERD are managed using a standard dose of PPI or potassium-competitive acid blockers in Korea. Additionally, most patients with GERD are unlikely to take medications continuously. Generally, the compliance rate of PPI is not high in patients with GERD. ${ }^{10}$ Therefore, the assumption that all patients will continue to take double dosage of PPI for approximately 10 years is unrealistic.

Nevertheless, anti-reflux surgery certainly appears to be a cost-effective option for patients who need PPI over a long period of time. Although study settings and assumptions varied across studies, many studies suggest 5-10 years as a break-even point for anti-reflux surgery over medication. ${ }^{11-14}$ If patients completely respond to PPI therapy but fail to discontinue the medication, antireflux surgery may enable the cessation of medication and thus, reduce ongoing costs. Patients who do not respond to PPI but are diagnosed with reflux-related diseases (ie, abnormal acid reflux despite PPI or reflux hypersensitivity) by systematic clinical work up including endoscopy, esophageal biopsy, esophageal manometry, and $\mathrm{pH}$ monitoring, may also benefit from anti-reflux surgery. In summary, for GERD patients managed by long-term medication, anti-reflux surgery may be a good treatment option, based on costeffectiveness.

\section{Financial support: None.}

\section{Conflicts of interest: None.}

\section{References}

1. Katz PO, Gerson LB, Vela MF. Guidelines for the diagnosis and management of gastroesophageal reflux disease. Am J Gastroenterol 2013;108:308-328.

2. Chiba N, De Gara CJ, Wilkinson JM, Hunt RH. Speed of healing and symptom relief in grade II to IV gastroesophageal reflux disease: a metaanalysis. Gastroenterology 1997;112:1798-1810.

3. Everhart JE, Ruhl CE. Burden of digestive diseases in the United States part I: overall and upper gastrointestinal diseases. Gastroenterology 2009;136:376-386.

4. El-Serag HB, Sweet S, Winchester CC, Dent J. Update on the epidemiology of gastro-oesophageal reflux disease: a systematic review. Gut 2014;63:871-880.

5. Chang FY. Variations in the reported prevalence of gastroesophageal reflux disease in Taiwan. J Chin Med Assoc 2012;75:91-92.

6. Spechler SJ, Hunter JG, Jones KM, et al. Randomized trial of medical versus surgical treatment for refractory heartburn. N Engl J Med 2019;381:1513-1523.

7. Park S, Park JM, Kim JJ, et al. Multicenter prospective study of laparoscopic Nissen fundoplication for gastroesophageal reflux disease in Korea. J Neurogastroenterol Motil 2019;25:394-402.

8. Park CH. Surgical treatment for gastroesophageal reflux disease: is it effective even for Asians? J Neurogastroenterol Motil 2019;25:337-339.

9. Park S, Park S, Park JM, et al. Anti-reflux surgery versus proton pump inhibitors for severe gastroesophageal reflux disease: a cost-effectiveness study in Korea. J Neurogastroenterol Motil 2020;26:215-223.

10. Van Soest EM, Siersema PD, Dieleman JP, Sturkenboom MC, Kuipers EJ. Persistence and adherence to proton pump inhibitors in daily clinical practice. Aliment Pharmacol Ther 2006;24:377-385.

11. Romagnuolo J, Meier MA, Sadowski DC. Medical or surgical therapy for erosive reflux esophagitis: cost-utility analysis using a Markov model. Ann Surg 2002;236:191-202.

12. Cookson R, Flood C, Koo B, Mahon D, Rhodes M. Short-term cost effectiveness and long-term cost analysis comparing laparoscopic Nissen fundoplication with proton-pump inhibitor maintenance for gastrooesophageal reflux disease. Br J Surg 2005;92:700-706.

13. Heudebert GR, Marks R, Wilcox CM, Centor RM. Choice of longterm strategy for the management of patients with severe esophagitis: a cost-utility analysis. Gastroenterology 1997;112:1078-1086.

14. Grant A, Wileman S, Ramsay C, et al. The effectiveness and costeffectiveness of minimal access surgery amongst people with gastrooesophageal reflux disease - a UK collaborative study. The REFLUX trial. Health Technol Assess 2008;12:1-181, iii-iv. 